\title{
INVESTIGAÇÃO SOBRE ATLETISMO DE COMPETIÇÃO PARA JOVENS COM SÍNDROME DE DOWN
}

\author{
RESEARCH ON ATHLETICS COMPETITION \\ IN YOUNG PEOPLE WITH DOWN SYNDROME \\ RECHERCHE SUR LA COMPETITION EN ATHLETISME \\ AVEC DES JEUNES PORTEURS DU SYNDROME DE DOWN \\ EXPERIENCIA ACERCA DE ATLETISMO DE COMPETICIÓN \\ CON JÓVENES CON SÍNDROME DE DOWN
}

Jesús Molina Saorín*

\section{RESUMO}

Nosso ponto de partida é o esporte de competição e, em concreto, a modalidade de Atletismo, incorporando o âmbito da Pedagogia e da Medicina como elementos chave para encontrar aqueles parâmetros de atuação que contribuam para a melhoria da autonomia, qualidade de vida e inserção sociolaboral das pessoas com sindrome de Down. Em nossa experiência de trabalho com um grupo de jovens com sindrome de Down na prática do atletismo de competição, podemos comprovar que são trabalhados os aspectos da autonomia, da autoregulação do próprio esforço ou da insistência, além da adaptação funcional, que, no nível do sistema cardiovascular, fica garantida, da mesma forma que para uma pessoa sem sindrome de Down submetida a um trabalho aeróbico. Portanto, trata-se de uma ótima contribuição, do ponto de vista da saúde da pessoa.

Palavras-chave: Atletismo de competição. Sindrome de Down. Autonomia. Qualidade de vida.

* Pós-Doutor em Pedagogia. Doutor em Pedagogia. Doutor Europeu em Pedagogia. Graduado em Pedagogia e Educação Física. Mestre em síndrome de Down. Professor do Departamento de Didáctica y Organización Escolar, Facultad de Educación, Universidad de Murcia (UMU - Espanha). Professor da Universidad Nacional de Ensino a Distancia (Uned - Espanha) (jesusmol@um.es, www.um.es/jesusmolina). 


\section{INTRODUÇÃO}

Neste trabalho situamo-nos, sem dúvida, ante uma aposta altamente inovadora. Isto ocorre uma vez que atuam em conjunto os seguintes elementos:

1) A consideração do desporto não como um fim em si, mas como um meio para conseguir não só a inserção social das pessoas com incapacidade psíquica (SORESI; NOTA, 2000), mas também a construção progressiva de uma imagem mais positiva de si mesmas.

2) O compromisso de propor e elaborar marcos teórico-práticos que sirvam como referência a futuras iniciativas, as quais possam ser levadas a cabo por instituiçôes interessadas na educação desportiva dos jovens com síndrome de Down.

3) O carácter transdisciplinar que impregna o desenvolvimento de todo o projeto ao longo de todas e cada uma de suas fases.

4) O fato de tratar-se de um projeto que reuniu o interesse e o compromisso das duas instituiçōes participantes (Fundown e Universidade de Múrcia).

\section{OBJETIVOS DO PROJETO FILÍPIDES}

O objetivo geral desta investigação é o seguinte: desenhar, desenvolver e avaliar um programa de treino desportivo na modalidade de atletismo de fundo, para jovens com sindrome de Down. Por outro lado, existe uma serie de objetivos específicos:

- criar e consolidar espaços de intercâmbio e desenvolvimento profissional entre os diferentes especialistas participantes no projeto;

- estabelecer canais de colaboração com as famílias dos atletas;

- analisar os contributos que se derivem com o objetivo de melhorar o programa de treino de atletismo e as suas sucessivas aplicações;

- identificar parâmetros médicos relacionados com a prática do atletismo com a finalidade de constatar as melhorias que se produzam na saúde como conseqüência direta da participação no programa de treinos;

- identificar aquelas estratégias do processo de ensino-aprendizagem, características da Educação Física e implicadas no programa de treino desportivo, com o objetivo de constatar as melhorias que se produzam, tanto no rendimento dos atletas no programa desenhado, como no seu desenvolvimento e crescimento pessoal.

\section{ESTADO ATUAL DO DESPORTO DIRIGIDO A PESSOAS COM SÍNDROME DE DOWN. O PROCESSO DE INVESTIGAÇÃO}

Consideramos oportuno dedicar um espaço a efetuar uma breve descrição do processo geral de investigação. Pensamos que esta tarefa pode contribuir para emoldurar convenientemente a informação e os resultados preliminares obtidos até o momento, aos quais dedicaremos um parágrafo específico. Nossa investigação discorre ao longo de quatro fases, as quais tratam de responder e dar cobertura ao seguinte objetivo geral de investigação: desenhar, desenvolver e avaliar um programa de treino desportivo na modalidade de atletismo de fundo, para jovens com síndrome de Down. 


\section{QUE FIZEMOS E DAÍ APRENDEMOS?}

No decorrer da primeira fase, e como primeira ação, constituiu-se o grupo transdisciplinar de investigação, o qual é integrado por profissionais do âmbito da medicina, da pedagogia e do desporto pertencentes às universidades de Málaga, de Múrcia e do Maranhão (Brasil). Do mesmo modo, o projeto conta com o assessoramento de atletas profissionais murcianos como D. Casiano Navarro, atleta de alta competição e sexta melhor marca mundial na modalidade de super-maratona. No que diz respeito à seleção dos participantes, é importante dizer que esta se organiza em torno da realização de provas médicas e de entrevistas com os candidatos e suas famílias. As provas médicas estão sendo levadas a cabo por professores experientes da Universidade de Málaga e da Universidade de Múrcia e pelos profissionais do Centro de Medicina Desportiva da Universidade de Múrcia. Em concreto, as provas médicas aludem a: história clínica, cineantropometria, exploração geral, exame de valoração da força, ecocardiologia, exploração do sistema cardiovascular, exploração do aparelho locomotor e teste de esforço em cicloergômetro.

A realização das entrevistas aos candidatos e suas famílias estruturaram-se em torno de dois focos de análise. Por uma parte, a obtenção de toda uma série de informações relacionadas com aspectos nutricionais e médicos, formando um dossiê que o candidato e sua família contribuíram para elaborar no dia da entrevista. Todas as entrevistas foram realizadas nos locais da Fundação Síndrome de Down e gravadas integralmente em áudio e vídeo. Para sua realização, elaboramos um guia de entrevista semi-estruturada que tratou de obter informação com respeito a três aspectos: conhecimento do projeto por parte do candidato e sua família; histórico desportivo do candidato; e conhecimento do candidato. A análise de conteúdo aplicado à transcrição das entrevistas propõe-nos os seguintes resultados, que, ainda que preliminares, contribuem para dar conta do estado atual do projeto Filípides.

Os pais e familiares dos candidatos consideram-se suficientemente informados a respeito dos objetivos que pretende conseguir o projeto e da responsabilidade que eles e seus filhos adquiririam em caso de ser selecionados. Neste sentido, destacam a utilidade que teve para eles a sessão informativa a que foram convocados, enfatizando a confiança que obtiveram ao poder dialogar com todos e cada um dos experientes integrantes do grupo de investigação. Em geral, detectamos uma valoração muito positiva do projeto por parte das famílias. Suas declarações afirmam a utilidade do projeto para seus filhos e a qualidade profissional dos especialistas e técnicos encarregados de levá-lo a cabo (VARELA; SARDINHA; PITETTI, 2001). A maior parte dos candidatos, rapazes e moças com síndrome de Down entre 14 e 30 anos, não parece ter um conhecimento preciso nem dos objetivos do projeto, nem dos grandes princípios em torno dos quais se articula. Esse desconhecimento parece estar mediatizado não só por sua maior ou menor capacidade de entendimento, mas também pela quantidade e qualidade da informação que suas famílias thes transmitiram (TSIMARAS et al., 2003). O espaço dedicado à realização das entrevistas foi aproveitado para informar convenientemente os candidatos, ou bem para clarificar as dúvidas que eles mesmos nos iam expondo. Do mesmo modo, pusemo-nos à sua disposição para marcar uma reunião individual com cada um deles, 
caso considerassem necessário. Em geral, os pais e familiares dos candidatos davam mostras de uma atitude de respeito para com seus filhos. Em nenhum caso observamos condutas que refletissem uma imposição ou pressão para que seus filhos participassem no projeto; pelo contrário, sempre apoiavam com maior ou menor interesse a decisão que seus filhos adotassem em caso de obter resultados positivos nas provas médicas. Em todos os casos, obtivemos uma resposta afirmativa ao perguntar-lhes sua disponibilidade para colaborar no projeto.

Esse dado é altamente positivo, já que, sem a colaboração e o envolvimento das famílias, resulta de todo impossível levar a bom termo a participação de seus filhos no projeto (SURAWY; ROBERTS; SILVER, 2005). No que respeita às questôes que abordavam diretamente o conhecimento do candidato, pudemos notar que as famílias sustentam uma concepção de seu filho/a que perpetua a imagem social (SOUSA, 2004) que tradicionalmente se construiu sobre as pessoas com síndrome de Down (simpático, afetuoso, bom, trabalhador...). Só quando mostramos nosso interesse em conhecer como eram seus filhos na realidade (TORRE, 2004), pudemos aceder a um conhecimento mais ajustado à realidade. Do mesmo modo, sempre que era possível, aproveitamos qualquer oportunidade para contrastar com os alunos o que seus pais e familiares diziam e pensavam deles. Nos situamos frente a um grupo de jovens que, em geral, vêem restringida sua interação social ao âmbito da família (LAFFERTY, 2005). Não costumam ter uma atitude de busca de amigos e costumam dedicar o seu tempo livre a ver TV, ou a estar em casa. Em geral, a maioria dos nossos candidatos praticaram um desporto em algum momento da sua vida. Só em alguns casos seguiu-se mantendo algum tipo de prática desportiva.

Para concluir este trabalho, não poderíamos deixar de fazer referência às competições realizadas pelos nossos atletas e à evolução verificada também nos tempos de corrida, no momento da prova.
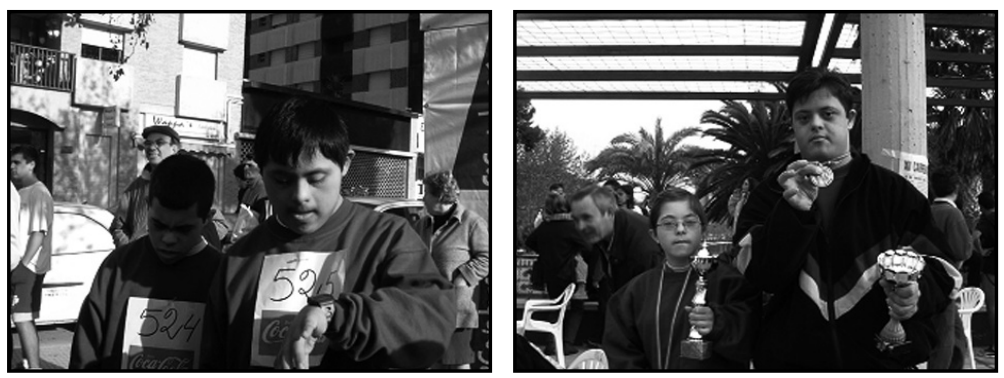

Depois de trabalhar com grande intensidade durante a primeira temporada, tudo parecia indicar que o grupo estava preparado para participar de uma competição regrada. No nosso trabalho, o significado da competição era muito importante e requeria uma reflexão ponderada sobre o que apreenderíamos com essa atividade (DODD; FLYNN, 2003). Por uma parte, a recompensa de participar de uma prova; por outra, a consciência de que, provavelmente, não íamos ganhar e, no entanto, nossa alegria por continuar 
superando nossas próprias marcas, nossos próprios medos, competição depois de competição, permite-nos qualificar essa experiência, sem sombra de dúvidas, como um verdadeiro sucesso em todas e cada uma das provas de competição que se foram sucedendo. Confiamos que a informação que pudemos difundir até o momento sobre o Projecto Filípides tenha conseguido cativar o interesse e o atendimento de todos aqueles profissionais da educação física e, em particular, daqueles que dedicam suas energias e ilusão ao trabalho com pessoas com maior ou menor incapacidade. Esperamos que a apresentação do projeto gere o necessário debate que, indubitavelmente, nos fará avançar, não só como pesquisadores, mas também como pessoas comprometidas com a mudança. Por meio da participação neste projeto, as pessoas com síndrome de Down desenvolvem uma multiplicidade de capacidades e habilidades sociais, num entorno totalmente normalizador e, por outro lado, contrário e fora de entornos segregadores. Sabemos que é uma tarefa difícil, e, muitas vezes, carregada de inúmeros obstáculos. No entanto, estamos totalmente convencidos da utilidade do nosso trabalho corroborado em todo momento pelo esforço e pela satisfação demonstrada pelos nossos atletas, e não só pelos resultados do ponto de vista físico e funcional demonstrados nesses dois anos. Eles e elas são, sem dúvida, os verdadeiros protagonistas deste trabalho.

\section{Referências}

DODD, Karen J.; FLYNN, Mark. A systematic review of the outcomes of cardiovascular exercise programs for people with Down syndrome. Archives of Physical Medicine and Rehabilitation, Indianapolis, IN, USA, v. 86, n. 10, p. 2.051-2.058, 2003.

LAFFERTY, Moira E. A stair-walking intervention strategy for children with Down's syndrome. Journal of Bodywork and Movement Therapies, Dublin, Ireland, v. 9, n. 1, p. 65-74, 2005.

SORESI, Salvatore; NOTA, Laura. A social skill training for persons with Down's syndrome. European Psychologist, Jena, Germany, v. 5, n. 1, p. 34-43, 2000.

SOUSA, Geida Maria Cavalcanti. Reflexōes do aprender a ser pesquisador na sua dimensão afetiva. Linhas Críticas, Brasília, Brazil, v. 10, n. 19, p. 267-280, jul./dez. 2004.

SURAWY, Christina; ROBERTS, Jill; SILVER, Amy. The effect of mindfulness training on mood and measures of fatigue, activity, and quality of life in patients with chronic fatigue syndrome on a hospital. Behavioural and Cognitive Psychotherapy, London, UK, v. 33, n. 1, p. 103-110, 2005.

TORRE, Saturnino de la. Educación emocional y estilos de vida. Linhas Críticas, Brasília, Brazil, v. 10, n. 19, p. 299-318, jul./dez. 2004.

TSIMARAS, Vassilis et al. Jog-walk training in cardiorespiratory fitness of adults with Down syndrome. Perceptual and Motor Skills, Missoula, MT, USA, v. 96, n. 32, p. 1.239-1.251, 2003.

VARELA, Ana Maria; SARDINHA, Luis Bettencount; PITETTI, Kenneth. Effects of an aerobic rowing training regimen in young adults with Down syndrome. American Journal on Mental Retardation, Wisconsin, WI, USA, v. 106, n. 2, p. 135-144, 2001. 


\section{Research on athletics competition in young people with Down syndrome}

\section{Abstract}

Our starting point is sports competition and, in particular, the modality of athletics, incorporating the fields of Pedagogy and Medicine as key elements to find out which parameters will contribute the most to improve the autonomy, quality of life and adaptation both social and to the labour market for people with Down Syndrome. Based on field work with youngsters with Down Syndrome that were practicing athletics competition, we can attest that sports has proved to be an excellent tool towards the autonomy and self-regulation of one's own effort or insistence, aspects dealt with in our work. Besides, functional adaptation at the level of the cardiovascular system works just the same as in people without Down Syndrome submitted to aerobic exercise. Therefore, this turns out to be a very good contribution, from the health point of view.

Keywords: Athletics competition. Down Syndrome. Autonomy. Quality of life.

\section{Recherche sur la compétition en athlétisme avec des jeunes porteurs du Syndrome de Down Résumé}

Notre point de partie est la compétition en sports et, en particulier, la modalité de l'athlétisme, incorporant les champs de la Pédagogie et de la Médecine comme éléments clefs pour trouver quels sont les paramètres qui contribuent le plus en vue de l'autonomie, la qualité de vie et l'adaptation tant sociale comme dans le marché du travail des personnes porteuses du Syndrome de Down. Comme base sur une recherche sur le champs avec des jeunes porteurs du Syndrome de Down qui pratiquaient la compétition en athlétisme, nous pouvons avancé que les sports ont démontré être un excellent instrument en vue de l'autonomie et l'auto-régulation de son propre effort ou insistance, aspects avec lesquels nous avons travailler. En plus, il a été démontré que l'adaptation fonctionnelle au niveau du système cardiovasculaire fonctionne de la même façon que chez les personnes sans le Syndrome de Down qui font des exercices aérobics. Donc, cela se révèle être une très bonne contribution du point de vue de la santé de ces personnes.

Mots clefs : Compétition en athlétisme. Syndrome de Down. Autonomie. Qualité de vie.

\section{Experiencia acerca de atletismo de competición con jóvenes con síndrome de Down} Resumen

Nuestro centro de partida es el deporte de competición y, más concretamente, la modalidad de atletismo, incorporando el ámbito de la Pedagogía y la Medicina como elementos clave a la hora de encontrar aquellos parámetros de actuación que contribuyan para la mejora de la autonomía, calidad de vida e inserción socio-laboral de las personas con sindrome de Down. Mediante nuestra experiencia de trabajo con un grupo de jóvenes con sindrome de Down practicando atletismo de competición, hemos podido comprobar que el trabajo de la autonomía, la autorregulación del propio esfuerzo y la constancia, han sido aspectos profundamente abordados y desarrollados en este estudio, además de la adaptación funcional que, a nivel del sistema cardiovascular, queda debidamente garantizada, al igual que sucede para una persona que, sin tener síndrome de Down, se somete a un programa de entrenamiento aeróbico. Por tanto, se trata de una contribución óptima desde el punto de vista de la salud de la persona.

Palabras-clave: Atletismo de competición. Sindrome de Down. Autonomía. Calidad de vida.

Recebida I ${ }^{\mathbf{a}}$ versão em: 05.01.2008

Aceita $2^{\text {a }}$ versão: 20.04 .2008 